\title{
Terahertz harmonic gyrotron based on spoof surface plasmon
}

\author{
Si-Qi Li \\ Department of Electronics, Peking \\ University \\ Beijing 100871, China
}

\author{
Zi-Wen Zhang \\ Department of Electronics, Peking \\ University \\ Beijing 100871, China
}

\author{
Chao-Hai Du* \\ Department of Electronics, Peking \\ University \\ Beijing 100871, China \\ *E-mail: duchaohai@pku.edu.cn \\ Fan-Hong Li \\ Department of Electronics, Peking \\ University \\ Beijing 100871, China
}

\author{
Juan-Feng Zhu \\ Department of Electronics, Peking \\ University \\ Beijing 100871, China
}

Adrian W. Cross

Department of Physics, University of Strathclyde

Scotland, British

\author{
$\mathrm{Pu}-\mathrm{Kun} \mathrm{Liu}$ \\ Department of Electronics, Peking University \\ Beijing 100871, China
}

\begin{abstract}
Gyrotron is based on the principle of relativistic electron cyclotron maser. With the working frequency increasing to terahertz band, the mode competition problem is caused by the interaction between harmonic cyclotron electrons and high order electromagnetic modes. In order to solve this problem, the interaction cavity of harmonic gyrotron based on spoof surface plasmon (SSP) is proposed in this paper, and its eigenmode frequency is studied, the concept of Band Gap is proposed. If the competition frequency is in the stopband, the competition mode will not be generated. Finally, the Particle-in-cell (PIC) simulation is carried out, which proves that the structure can make the terahertz gyrotron work in the single mode in state of high harmonics.
\end{abstract}

Index Terms - Terahertz, gyrotron, higher harmonic, spoof surface plasmon, mode competition

\section{INTRODUCTION}

Gyrotron, based on the principle of relativistic electron cyclotron maser, can generate high-stability coherent electromagnetic radiation in millimeter wave and terahertz frequency band. It can be used in plasma heating, imaging of dynamic nuclear polarization nuclear magnetic resonance, and high-speed wireless communication [1][2].

When the frequency of gyrotron reaches terahertz band, the interaction cavity of gyrotron generally adopts high-order electromagnetic mode. In order to reduce the intensity of magnetic field, the terahertz gyrotron usually works in the state of high harmonics. Therefore, it leads to the problem of mode competition. The same cyclotron harmonic may excite multiple modes with similar working frequency; at the same time, different cyclotron harmonics may excite different modes, and the frequency of the competing mode is approximately the frequency doubling relationship [3]. The excited competition mode will reduce the working efficiency of the gyrotron, seriously affect the working stability, and generate incoherent electromagnetic radiation.
In order to suppress the mode competition, the interaction cavity of harmonic gyrotron based on spoof surface plasmon is proposed in this paper. The characteristic of its eigenfrequency distribution is studied, and the possibility of the gyrotron working in the single mode in state of high harmonics is explored.

\section{BAND-GAP OF Spoof SuRface Plasmon Resonate CAVITY}

Spoof surface plasmon (SSP) was first proposed by a team led by J. Pendry of Imperial College London. They found that the structure of one-dimensional metal groove can reduce the metal plasma frequency $\omega_{p}$ to microwave band, so that this spoof structure can produce surface plasmon polaritons similar to the light wave band [4].

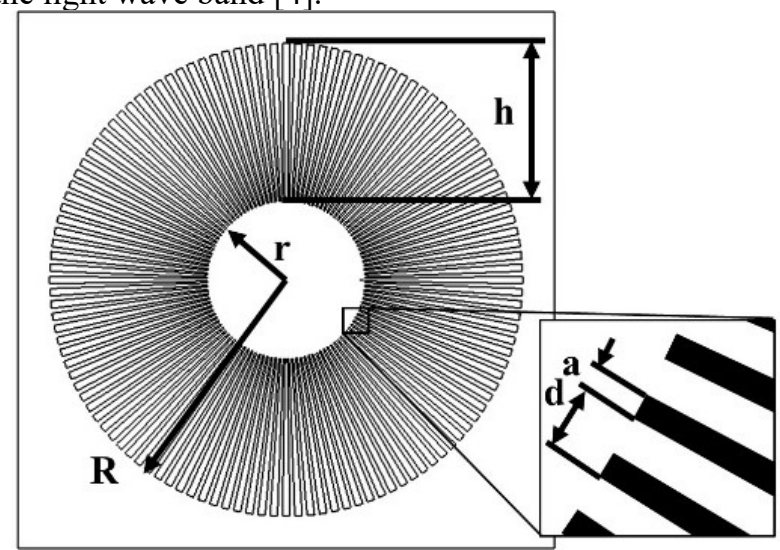

Fig. 1 The structure of the spoof surface plasmon resonator.

A resonate cavity based on spoof surface plasmon has been proposed (Fig. 1), which is a kind of closed textured cavity. The material of the outer wall of the resonant cavity is perfect electric conductor (PEC), and the groove with the depth of $\mathrm{h}=\mathrm{R}-\mathrm{r}$ along the radial direction of the circumference is made, $\mathrm{R}$ is the radius of the outer wall of the resonator, $r$ is the radius of the air cavity, 
and the material of the giant groove is perfect electric conductor (PEC). The grating constant is $d$, the duty cycle of grating is $a / d$, and the number of grating is $N$, which satisfies the quantitative relationship $N d=2 \pi r$. According to the approximate condition proposed by Pendry [4], the grating constant satisfies the subwavelength limit $d \ll \lambda_{0}$, and $\lambda_{0}$ is the wavelength corresponding to the eigen frequency of the resonator. The specific parameters are as follows: $N=120, a / d=0.6, r=3 R$, $r=0.5 \mathrm{~mm}$. In the analysis of eigenmodes, only the plane structure is considered, and the number of axial modes is not considered, or the electromagnetic wave is considered to be uniformly distributed along the axial direction.

The analytical solution of the eigenmode of the section of spoof surface plasmon resonator is obtained from [5]. Because this structure is circular symmetric, it is convenient to use polar coordinates.

$$
\frac{a}{d} \sin c^{2}\left(\frac{m a}{2 r}\right) \frac{J_{m}\left(k_{0} r\right)}{J_{m}^{\prime}\left(k_{0} r\right)} \frac{f}{g}=n_{g}
$$

Where $f=Y_{1}\left(k_{0} n_{g} r\right) J_{1}\left(k_{0} n_{g} R\right)-Y_{1}\left(k_{0} n_{g} R\right) J_{1}\left(k_{0} n_{g} r\right)$, $g=Y_{1}\left(k_{0} n_{g} R\right) J_{0}\left(k_{0} n_{g} r\right)-Y_{0}\left(k_{0} n_{g} r\right) J_{1}\left(k_{0} n_{g} R\right)$. This is a transcendental equation. $J_{0}$ and $J_{1}$ are the zero order and the first order forms of the first kind of Bessel function respectively. $Y_{0}$ and $Y_{1}$ are the zero order and the first order forms of the second kind of Bessel function, respectively. Different $N$ represents different modes. Through observation, we can find that the eigen equation contains the frequency of resonance.

Because the principle of gyrotron is the interaction between the gyrotron electron beam and the transverse electric field to exchange energy, we mainly consider TE mode here. The eigen frequencies of $\mathrm{TE}_{\mathrm{n}, 1} 、 \mathrm{TE}_{\mathrm{n}, 2} 、 \mathrm{TE}_{\mathrm{n}, 3}$ mode are solved and plotted on a two-dimensional coordinate graph. (Fig. 2.)

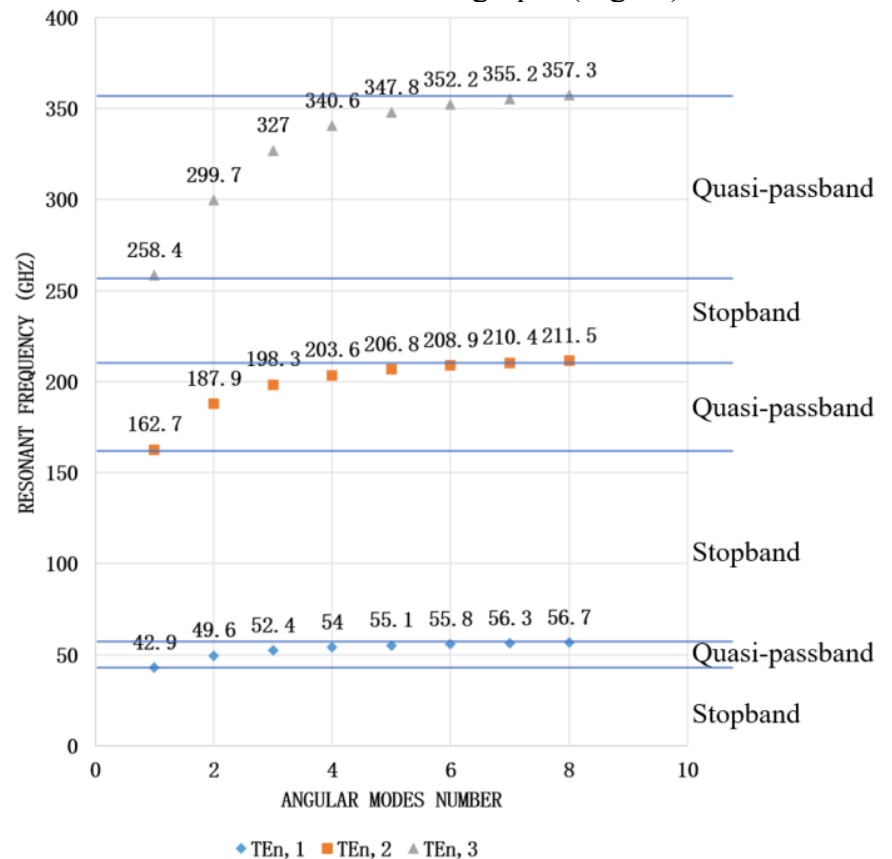

Fig. 2 The resonant frequency of $\mathrm{TE}_{\mathrm{n}, 1}, \mathrm{TE}_{\mathrm{n}, 2}, \mathrm{TE}_{\mathrm{n}, 3}$

It can be seen that for the same radial mode, the larger the number of angular modes is, the higher the resonance frequency is, the smaller the resonance frequency gap between adjacent modes is, and finally converges to a frequency value [6]. For different radial modes, the larger the number of radial modes is, the higher the resonance frequency is. There is no resonance frequency between $0-42.885 \mathrm{GHz}$, so $0-42.885 \mathrm{GHz}$ can be called "first stopband". The resonant frequency of $\mathrm{TE}_{1,1}, \mathrm{TE}_{2,1}$, $\mathrm{TE}_{3,1}, \mathrm{TE}_{4,1} \cdots \cdots \mathrm{TE}_{\mathrm{n}, 1}$ mode is only between $42.885-60 \mathrm{GHz}$, so we can call $42.885-60 \mathrm{GHz}$ as "first quasi-passband". There is no resonance frequency between $60-162.662 \mathrm{GHz}$, so 60$162.662 \mathrm{GHz}$ can be called "second stopband". The resonant frequency of $\mathrm{TE}_{1,2}, \mathrm{TE}_{2,2}, \mathrm{TE}_{3,2}, \mathrm{TE}_{4,2} \cdots \cdots \mathrm{TE}_{\mathrm{n}, 2}$ mode is only between $162.662-220 \mathrm{GHz}$, so we can call $162.662-220 \mathrm{GHz}$ as "second quasi-passband". There is no resonance frequency between $220-258.386 \mathrm{GHz}$, so $220-258.386 \mathrm{GHz}$ can be called "third stopband". The resonant frequency of $\mathrm{TE}_{1,3}, \mathrm{TE}_{2,3}, \mathrm{TE}_{3,3}$, $\mathrm{TE}_{4,3} \cdots \cdots \mathrm{TE}_{\mathrm{n}, 3}$ mode is only between $258.386-370 \mathrm{GHz}$, so we can call 258.386-370 GHz as "third quasi-passband". We can call this phenomenon as Spoof Surface Plasmon "Band Gap". The "quasi-passband" and "stopband" are shown in Figure 2.

Compared with a traditional circular waveguide resonator, the spoof surface plasmon resonator has two advantages. First, there is "stopband". Because the resonant frequency of each mode of the section of the spoof surface plasmon resonator is the cut-off frequency of each mode of the spoof surface plasmon resonator, and the gyrotron works in the near cut-off area, so the working frequency of the gyrotron is similar to which of the section of the spoof surface plasmon resonator. If the competition frequency of the gyrotron is in the "stop band" of the resonance frequency, the mode competition can be eliminated fundamentally. However, there is no "stopband" in the circular waveguide. The distribution of the eigenfrequency is related to Bessel function. There are eigenmodes near any frequency point. Second, for the same radial number, when the angular number $\mathrm{n}$ is small, the interval between adjacent eigenfrequencies is large, so the mode competition problem can be reduced by setting the operating frequency at the frequency point of the angular number $\mathrm{n}$ is small.

\section{Terahertz HARMONIC GyRotron BASED ON SPOOF SURFACE PLASMON}

Next, we design a terahertz harmonic gyrotron based on spoof surface plasmon. The working mode of the gyrotron is $\mathrm{TE}_{2,3}$, the operating frequency is $299.749 \mathrm{GHz}$ and the electron cyclotron frequency is $74.94 \mathrm{GHz}$. Therefore, it works in the fourth harmonic state, which is unprecedented in all previous studies. There are two advantages of this design. First, the fundamental frequency of electron cyclotron radiation is $74.94 \mathrm{GHz}$, the second harmonic frequency is $149.87 \mathrm{GHz}$, and the third harmonic frequency is $224.81 \mathrm{GHz}$. These three frequencies are competitive frequencies, but they are all in the "stopband" frequency. There is no corresponding eigenmode in the spoof surface plasmon resonator, and each of competitive lower-order harmonics cannot find an available mode in the stopbands and accordingly, cannot start oscillate. Second, the operating frequency is $299.749 \mathrm{GHz}$, and the competition modes near this frequency are $258.386 \mathrm{GHz} \mathrm{TE}_{1,3}$ mode and 327.017GHz $\mathrm{TE}_{3,3}$ mode, but the frequency interval is far, so it 
is difficult to compete with the operating frequency. The specific design parameters are shown in Table 1.

TABLE 1 Design parameters of terahertz harmonic gyrotron

\begin{tabular}{ll}
\hline Parameters & value \\
\hline Emitting voltage & $1 \mathrm{kV}$ \\
Emitting current & $50 \mathrm{~mA}$ \\
Operating frequency & $299.749 \mathrm{GHz}$ \\
Electron cyclotron frequency & $74.94 \mathrm{GHz}$ \\
Harmonic number & 4 \\
Guiding radius & $0.35 \mathrm{~mm}$ \\
Ratio of transverse to longitudinal speed & 2 \\
Operating mode & $\mathrm{TE}_{2,3}$ \\
Cavity length & $5 \mathrm{~mm}$ \\
Internal radius & $0.5 \mathrm{~mm}$ \\
External diameter & $1.5 \mathrm{~mm}$ \\
Applied magnetic field & $2.78 \mathrm{~T}$ \\
\hline
\end{tabular}

By using the Particle-in-cell (PIC) module of CST particle studio, the interaction process of electron beam and electromagnetic field in the harmonic gyrotron resonator based on spoof surface plasmon is simulated. Figure 3 shows the frequency spectrum of the field in the cavity. It shows that there is only one frequency in the spoof surface plasmon resonator, and there is no competition frequency. Because of the fourth harmonic working state, the efficiency is inevitably low. The electron emission power is $50 \mathrm{~W}$, the output power is $2.8 \mathrm{~mW}$, and the efficiency is $0.0056 \%$.

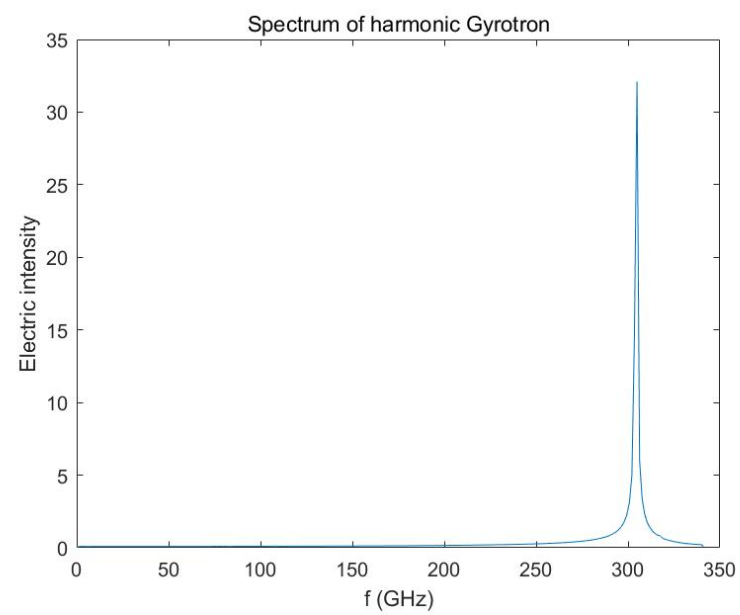

Fig. 3 Frequency spectrum of field in resonator

\section{CONCLUSION}

The results show that the eigenfrequencies of the section of the spoof surface plasmon resonator have "quasi-passband" and "stopband", which provides a way to reduce the mode competition of the harmonic gyrotron. Finally, the simulation results show that the terahertz gyrotron can work in the single mode in the state of high harmonics by using the spoof surface plasmon resonator, which greatly reduces the requirement of magnetic field.

\section{ACKNOWLEDGMENT}

This work was supported in part by the National Natural Science Foundation of China under contracts 61861130367, 61531002, NSAF-U1830201. It was also supported in part by the Newton Advanced Fellowship from Royal Society (NAF/R1/180121), United Kingdom.

\section{REFERENCES}

[1] Chu, and R. K., "The electron cyclotron maser," Reviews of Modern Physics, vol. 76, no. 2, pp. 489-540, 2004.

[2] Nusinovich, Gregory S. and Thumm, Manfred K. A. and Petelin, Michael I., "The Gyrotron at 50: Historical Overview," Journal of Infrared Millimeter \& Terahertz Waves, vol. 35, no. 4, pp. 325-381, 2014.

[3] Kao, S. H. and Chiu, C. C. and Pao, K. F. and Chu, K. R. "Competition between Harmonic Cyclotron Maser Interactions in the Terahertz Regime," Physical Review Letters, vol. 107, no. 13, p. 135101, 2011.

[4] Pendry J B, Martin-Moreno L and Garcia-Vidal F J., "Mimicking Surface Plasmons with Structured Surfaces," Science, Vol. 305, No. 5685, pp. 847-848, 2004.

[5] Xu, Bingzheng, et al., "Multiband localized spoof plasmons in closed textured cavities," Applied Optics, vol. 53,no. 30, p. 6950, 2014.

[6] Liao, Zhen , et al., "High-order localized spoof surface plasmon resonances and experimental verifications," Scientific Reports, vol. 5, 2015 . 